\title{
Qos-Aware Dynamic Composition of Web Services Using Novel Planning Based Approach
}

\author{
${ }^{1}$ N. Mohamed Yasin \\ ${ }^{1}$ Department of Math and IT Department \\ Center for Preparatory Studies, Sultan Qaboos University Muscat, \\ Sultanate of Oman.
}

\begin{abstract}
Nowadays, web services are essential to all organization to build their business. But the major issue in web service is quality of service. Web service does not allow multiple processes in parallel with effective quality of service measures. In this paper, I suggest a novel planning-based approach for service composition instead of predefined workflow model. With the help of numerical and temporal features, the novel planning-based approach converts QoS-aware composition task for a planning problem automatically. Of an edge, with numerical optimization and logical reasoning to handle complex temporal planning problems, I have used existing one and a self-developed one including the state-of-the-art planners. While fulfilling different global QoS constraints, the outlook can find an optimal overall QoS value with composite service graph. With the help of large web service repositories, I have conducted great experiments on implementing it in a prototype system.
\end{abstract}

Keywords: QoS, Temporal Planning, Novel planning. 


\section{INTRODUCTION}

Web services are self- contained, modular, selfdescribing, platform independent components of software over the Internet that are published with the help of service providers. Many organizations keep their fundamental business, except outsource of other application services that have been published over the Internet since the availability of the web services. Allowing the sharing and construction of autonomous and independent software has been widely applied by web service composition (WSC). According to achieve the objective of user defined the computer program that can automatically integrate, select and multiple invoking on regarding the web services. By the requirement to improvise the efficiency and efficacy of integrating the web services, the lot of web services automated WSC and service multipliers gets motivated.

Execution price, execution success rate, reputation and the availability belongs to the group of non-functional properties of QoS which has been widely used to represent it and compare them for composition of QoS services provided by the similar functionality. While all the QoS constraints are satisfied the QoS is said to be optimal regarding the user preferential and the global constraints. The web services can deploy its message or function to the rest of the world according to the availability of services provided over the web service repositories. While the exchange of data between the different platforms and different applications has been employed over the composition of web services.

\section{RELATED WORKS}

In recent times, lot of work that had been carry out in the field of web services. Many have conducted experiments and approached to make an advancement in the research over increasing the quality of service in web services. Accordingly, to understand the different and various approaches proposed by different authors and researchers in the field of QoS particularly in web services is taken as a review work here.

In this article [1], the author proposed from their workflow and research community of AI planning for an overview made on the recent research efforts of automated web service composition. The web services are built and updated on the fly. To generate the composition plan manually and the human ability to analyze them is beyond already. Most of the approaches have been applied to resolve that problem and mostly are inspired by the authors in crossenterprise on AI planning and workflow. The advantage mainly taken here by the author is it generates the composition plan manually and main disadvantage is it does not cost effective and also it arises a lot of compensations to QoS metrics. The QoS-aware selection algorithm for automate transactional TQoS and web service composition is proposed by another author [2]. An important challenge of meeting the user requirements and for each activity of workflow is important on selection of web services. Some challenge research issues that has been brought by the service oriented architectures are mostly implemented by the web services. Indeed, among a group of them that satisfies some functionalities is a complex task in the selection of one Web service, generally depends on a combined evaluation of QoS. The composition of web services that its capability to construct recursively the workflow of composite web services and other existing web services that are developed by different organizations and its offers diverse functionalities (e.g. Payment, ticket purchase), Quality of Service (QoS) values (e.g., success rate, execution price) and transactional properties 
(e.g., composition or not). This survey [2] addresses the composition and selection of web services by their transactional properties TQoS that satisfies the user preferences conveyed as weights over QoS and as risk levels explaining significant transactional requirements. This considers user's preferences as global manner and they do not meet user's requirements successfully is the disadvantage on this paper. The much needed research is regarding the AI based planning on automated Web Service Composition (WSC) that is done by offline prior for its execution. This explained or surveyed by the paper [3] PreferenceBased Web Service Composition. On investigating the preference-based Task Network WSC system of Hierarchical, the resultant is performed in a semantic web employment. To optimizing the QoS, user preferences and trust convinced the WSC on recent researches. On attempting an online information gathering and the balance trade-off offline composition that produce without excessive data gathering and high-quality compositions efficiently while testing the class of WSC problems. While some of this optimizations are data-dependent, can be interesting, useful and can be done offline, the execution atlas for some gathering services over information. This paper illustrates the potential improvement in speed and quality of composition generation by this approach. The major advantages as in this paper [3] is it uses semantic web employing and its disadvantage results it does not cost effective and also it arises a lot of compensations to QOS metrics. In paper [4] that has been surveyed for Web Service Composition of middleware functionalities. It helps service providers to maintain their identity functionality. The formation of Business- toBusiness (B2B) collaborations creates unprecedented opportunities by the web services that are driven by the paradigmatic shift from internet of programmatic interactions to Web of manual interactions. The main disadvantage here is QoS metric point's user specification also service provider profit maximization, but this focus only service providers. A middleware platform that conveys this issue of selecting Web services for the need of their composition in a way that maximizes user satisfaction over QoS attributes by the structure of the composite service, while satisfying the constraints set by the user. One based on global allotment of tasks to services and the other based on (tasklevel) local service selection using integer programming are compared and described as the two selection approaches. A significant momentum is gained by the existing ones by the composition of creation of valueadded services in particular. A web service provides identical functionality or overlapping with the QoS needs a choice that determines the services to participate in a given composite task. In the existing system, the author proposed a predefined workflow which refers to a set of tasks. For every individual task, each of them performs a task that correspond a group of candidate web services. This explains the workflow model with task for candidate services. Based on the conventional approaches, predefined work flow reduces the search space into a smaller one. Thus it results into two illustrations. One is that for composition task, these proposals do not guarantee to find a solution that satisfies the QoS constraints globally that exists under a different workflow.

Another is that they can't make the overall QoS an optimal while compared with workflows on others. The disadvantages in the existing system results, in particular, there has been a huge search space while the number of Web services results large search space. For multiple workflows, most of existing QoS metrics is restricted. The problem definition to resolve in the proposed system includes existing QoS-aware WSC approaches fall short with globally optimal QoS on finding solutions, because it is a very complex optimization problem with, discrete decisions, logical reasoning, numerical optimization, and temporal constraints. The number of services over internet becomes large because of their huge search space. As a conclusion, for predefined workflows, most existing QoS-aware WSC methods gets restricted in particular. 


\section{PROPOSED ARCHITECTURE}

In this paper, I have yet to propose that the QoS optimization over WSC by the novel planning-based approach. While satisfying the particular global QoS constraints, one can specify multiple QoS constraints globally and their regarding user preferences. Our proposal optimizes the overall QoS and finds composite services. I hold advancement in the numerical temporal planning to solve the resultant planning problems efficiently and optimize by our temporal numerical planner. With the help of numeric and temporal features, our approach changes the preferences to a planning problem and multiple global QoS constraints of the composition task. The Proposed Approach supports multiple work flow model.

\subsection{Architecture: Dynamic Composition of Web Services}

As I proposed in the Fig.1, the user request to the QoS metrics and the specification detail will take into a main consideration of the constraints.

Then, it gathers the success ratio of the metrics and the specification details of the user request. The followed request has been resolved into a response if the success ratio meets the needs of the end user if not the analyzed suggestion is followed to previous phases and produces the required suggestion at the time of no case availability of the user preferences.

While the conventional approaches, only our approach can optimize the QoS and finds the optimal QoS under a predefined workflow.

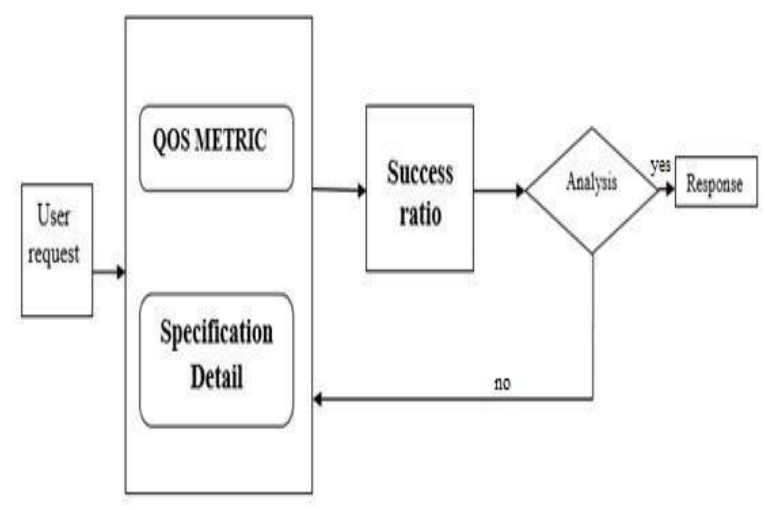

\section{Fig.1: Architecture-Dynamic Composition of}

\section{Web Services}

\subsection{Optimization Algorithm}

Input : Specification Details 
$8^{\text {th }}$ International conference on Research in Engineering, Science and Technology
November 2-4, 2018

Output: suggestions through WSDL

Request=getUserRequest (request);

While(isRequest)

\{

For each is select request details

\{

Arraylist arraylist; Foreach (read_metrics)

\{

Getavailability; If(is_available)

\{

Marks_its_booked;

\}

else

\{

Getmetrics_user_request; Foreach(getavailability based on metrics) \{

Arraylist.store(items);

\}

\}

\}

SendStoreditems;

\} $\}$

\subsection{Metric learning Algorithm}

Pseudocode: Service side:

getParameter (user_request) select_requested_item; check availability;

if(available) $\{\quad$ make its selected

\} else

\{

Get_metrics_of_user request Search database with metrics If (Dbcontent: available)

\{

Store_data in arrayList

\}

else

\{

Return "no data found..."

\}

Sort Arraylist based on metrics percentage Return arraylist 
\}

\subsection{Modules}

In the above figure Fig.2., the modules are the main functional block. The composition of Web Services will be done by these modules.

- Service

- Preferences

- Numerical Planning

- Similar Metrics

- Client

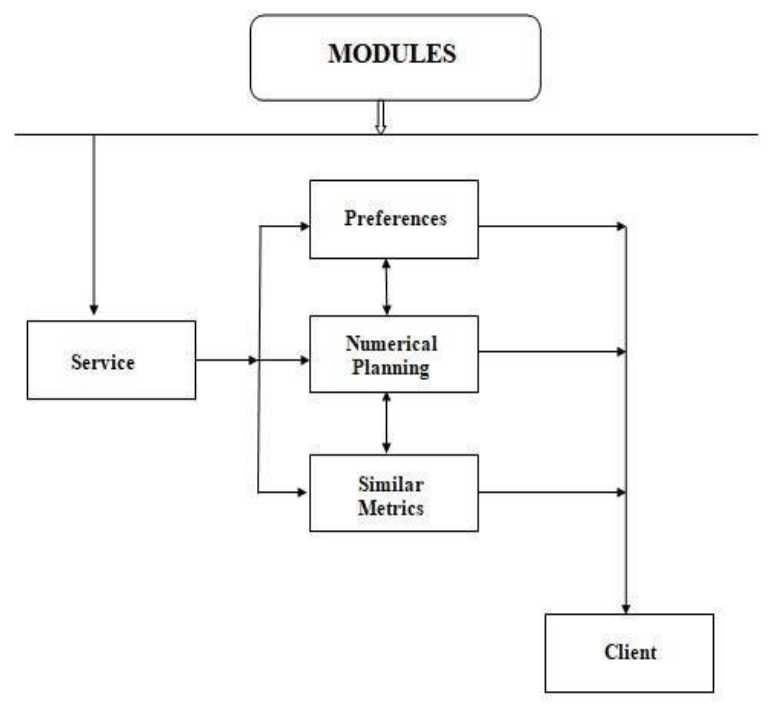

\section{Fig.2. Modules}

\subsubsection{Service}

Service contains list of codes that need to generate WSDL (Web Service

\section{Description}

Language) document and client access. Client can access the application based on what I write in the service code. In service, I need to mention list of services and its limitation.

\subsubsection{Preferences}

The Preferences have the details about priority of services because sometimes two or more services running at the same time, so I need to give the priority to services based on its process, execution time and etc., this preferences section contains these details to avoid deadlock conditions. 


\subsubsection{Numerical Planning}

This numerical planning method consists of the information about the process such as execution time, probability of success, process metrics. By using these details, I can make suggestion when no availability status.

\subsubsection{Similar Metrics}

In this module, execution begins when the process cannot be success or no availability status. According to this process, it retrieves metrics of requested process and this find similar process from the availability.

\subsubsection{Client}

The Client will access the services provided by the WSDL document. Client is an end user of this project. The advantage of this web service concept is service and client can be different language.

\section{CONCLUSION}

In this paper, I have implemented web service composition to produce availability with multiple user specifications. Also this system produces suggestion when no availability found for entered specifications. According to our project scope, this system achieves QoS metrics with price, availability and possibility of success. The number of services provided by web becomes less because of their reduced search space. As an outcome for predefined workflows, the existing QoS-aware WSC methods are not restricted in particular.

In future process, I will try to implement some other quality measures that based on the current trends. Also, I can try in automotive planning for a kind of quality of service.

\section{REFERENCES}

[1] J. Rao and X. Su, "A survey of automated Web service composition methods," Lecture Notes in Computer Science (LNCS), vol. 3387, pp.

43-54, 2005.

[2] J. Haddad, M. Manouvrier, and M. Rukoz, "TQoS: Transactional and QoS-aware selection algorithm for automatic Web service composition," IEEE Transactions on Services Computing (TSC), vol. 3, no. 4, pp. 73-85, 2010. 
[3] S. Sohrabi and S. Mcllraith, "Preference-based web service composition: A middle ground between execution and search," in Proceedings of International Semantic Web Conference (ISWC), 2010.

[4] L. Zeng, B. Benatallah, A. Ngu et al., "QoS- aware middleware for Web services composition," IEEE Transactions on Software Engineering (TSE), vol. 30, no. 5, pp. 311-327,2004.

[5] D.A. Menasc'e, “Composing Web services: A QoS view," IEEE Internet Computing, vol. 8, no. 6, pp. 88-90, 2004.

[6] M. Jaeger, G. Rojec-Goldmann, and G.M' uhl, "QoS aggregation for Web service composition using workflow patterns," in Proceedings of International Enterprise Distributed Object Computing Conference, 2004.

[7] W. Zhang, Y. Yang, S. Tang et al., "QoS- driven service selection optimization model and algorithms for composite Web services," in Proceedings of Annual International Computer Software and Applications Conference (COMPSAC), 2007.

[8] B. Wu, C. Chi, and S. Xu, "Service selection model based on QoS reference vector," in Proceedings of IEEE Congress on Services, 2007.

[9] S. Hwang, E. Lim, C. Lee et al., "DynamicWeb service selection for reliable Web service composition," IEEE Transactions on Services Computing (TSC), vol. 1, no. 2, pp. 104- 116,2008.

[10] M. Falou, M. Bouzid, A. Mouaddib et al., "A distributed planning approach for Web services composition," in Proceedings of International Conference on Web Services (ICWS),2010.

[11] P. Bertoli, R. Kazhamiakin, M. Paolucci et al., "Continuous orchestration of Web services via planning," in Proceedings of International Conference on Automated Planning and Scheduling (ICAPS), 2009.

[12] J. Hoffmann, P. Bertoli, M. Helmert et al., "Message-based Web service composition, integrity constraints, and planning under uncertainty: A new connection," Journal of Artificial Intelligence Research (JAIR), vol.

35, no. 1, pp. 49-117, 2009.

[13] S. Oh, D. Lee, and S. Kumara, "Effective Web service composition in diverse and large-scale service networks," IEEE Transactions on Services Computing (TSC), vol. 1, no. 1, pp. 15-32, 2008.

[14] X. Zheng and Y. Yan, "An efficient syntactic Web service composition algorithm based on the planning graph model," in Proceedings of International Conference on Web Services (ICWS), 2008. 
$8^{\text {th }}$ International conference on Research in Engineering, Science and Technology
November 2-4, 2018

[15] J. Hoffmann, P. Bertoli, and M. Pistore, "Web service composition as planning, revisited: In between background theories and initial state uncertainty," in Proceedings of AAAI Conference on Artificial Intelligence (AAAI), 2007. 